\title{
A QCD Calculation of the Interaction of Quarkonium with Nuclei
}

\author{
Michael Luke, Aneesh V. Manohar, and Martin J. Savage \\ Department of Physics 0319 \\ University of California, San Diego \\ 9500 Gilman Drive \\ La Jolla, CA 92093-0319
}

\begin{abstract}
The interaction of quarkonium with nuclei is studied in the $m_{Q} \rightarrow \infty$ limit of QCD, where the binding energy is found to be exactly computable. The dominant contribution to the interaction is from two-gluon operators. The forward matrix elements of these twogluon operators can be determined from the QCD scale anomaly, and from deep inelastic scattering. We apply our results to the $\Upsilon$ and $J / \psi$, treating the $Q \bar{Q}$ interaction as purely Coulombic. We find the $\Upsilon$ binds in nuclear matter with a binding energy of a few $\mathrm{MeV}$, while for the $J / \psi$ binding is of order $10 \mathrm{MeV}$. For the $J / \psi$ in particular we expect confinement effects to produce large corrections to this result.
\end{abstract}

UCSD/PTH 92-12

March 1992 
There has been some recent interest in the possibility of bound states occurring between quarkonium and nuclei [1] [2]. By scaling $K$-nucleon scattering from high energies and modifying the interaction due to the reduced size of charmonium the binding energy of the $\eta_{c}$ in nuclear matter [2] is estimated to be $\sim 30 \mathrm{MeV}$. The motivation for studying the interactions between quarkonium and nuclei is clear. The interactions of nuclei cannot be computed starting directly from the QCD Lagrangian. However, they can be parameterised by using a phenomenological Lagrangian with nucleon and meson degrees of freedom. Thus a study of nuclear forces provides some understanding of the dynamics of nucleons and the pions which are produced by the spontaneous breaking of chiral symmetry. The interactions of quarkonium allows us to study a different aspect of QCD, the interactions of gluons. The most significant difference between the nucleon-nucleon potential and a quarkonium-nucleon potential is that there is no valence light quark exchange in the latter. The coupling constants of the interactions between quarkonium with pions, nucleons and other light states arise solely from gluon induced interactions.

In this paper, we study the interactions of a $Q \bar{Q}$ bound state with nuclear matter, in the limit that the mass $m_{Q}$ of the quark, and therefore the inverse radius $r_{Q}^{-1} \sim \alpha_{s}\left(r_{Q}^{-1}\right) m_{Q}$ of the $Q \bar{Q}$ bound state, is much larger than the QCD scale $\Lambda_{\mathrm{QCD}}$. We then apply this to the cases of experimental interest, the $\bar{c} c$ and $\bar{b} b$ bound states, and calculate their binding energies in nuclear matter.

At scales $\mu \ll r_{Q}^{-1}$, the $Q \bar{Q}$ bound state looks like a colour singlet, pointlike object. Its dynamics will be described at low momenta by an effective Lagrangian in which nonrenormalizable terms are suppressed by powers of the compositeness scale $\Lambda_{Q}=r_{Q}^{-1}(\simeq$ $\alpha\left(\Lambda_{Q}\right) m_{Q}$ for a Coulomb bound state). The gauge invariant gluon couplings of lowest dimension are the dimension 7 operators

$$
\begin{aligned}
& \mathcal{L}_{\mathrm{int}}=\sum_{v} \frac{1}{\Lambda_{Q}^{3}}\left(P_{v}^{\dagger} P_{v}-V_{\mu v}^{\dagger} V_{v}^{\mu}\right)\left(c_{E} \mathcal{O}_{E}+c_{B} \mathcal{O}_{B}\right) \\
& \mathcal{O}_{E} \equiv-G^{\mu \alpha A} G_{\alpha}^{\nu A} v_{\mu} v_{\nu}, \quad \mathcal{O}_{B} \equiv \frac{1}{2} G^{\alpha \beta A} G_{\alpha \beta}^{A}-G^{\mu \alpha A} G_{\alpha}^{\nu A} v_{\mu} v_{\nu},
\end{aligned}
$$

where $P_{v}$ and $V_{v}^{\mu}$ create pseudoscalar $\left(\eta_{c}\right.$ or $\left.\eta_{b}\right)$ and vector $(J / \psi$ or $\Upsilon)$ mesons respectively, with four velocity $v^{\mu}$. All the sensitivity to the detailed structure of the meson is encoded in the coupling constants $c_{E}$ and $c_{B}$, which must be calculated from the underlying QCD dynamics.

1 This is a just a covariant formulation of the leading terms in the usual multipole expansion[3]. 
Some explanation of (1) is in order. Since the interaction of a heavy state with light degrees of freedom does not change the velocity of the heavy state in the $m_{Q} \rightarrow \infty$ limit, the velocity is conserved, and it is convenient to describe mesons with different velocities by distinct fields [ [ [ The fields $V_{v}^{\mu}$ and $P_{v}$ are related to the standard fields $P$ and $V^{\mu}$ by the field redefinitions

$$
V_{v}^{\mu}(x)=\sqrt{2 m} e^{i m v_{\alpha} x^{\alpha}} V^{\mu}(x), \quad P_{v}(x)=\sqrt{2 m} e^{i m v_{\alpha} x^{\alpha}} P(x),
$$

where $m$ is the meson mass, and physical vector states satisfy $v_{\mu} V_{v}^{\mu}=0$. Writing the momentum as

$$
p^{\mu}=m v^{\mu}+k^{\mu},
$$

we see that derivatives acting on the redefined fields bring down factors of the small "residual" momentum $k^{\mu}$, so the derivative expansion does not contain potentially troublesome terms proportional to $m v^{\mu} / \Lambda_{Q}$. Also, in the heavy quark limit, the quark spin decouples and the resulting spin symmetry [5] forces the masses and couplings of the pseudoscalar and vector bound states to be equal (the minus sign in (1) is because a physical vector particle has spacelike polarization). There is also no term which converts a pseudoscalar to a vector at leading order in $1 / m_{Q}$. Hence, in the $m_{Q} \rightarrow \infty$ limit, the binding to nuclei of a vector meson is equal to that of the corresponding pseudoscalar meson.

In the rest frame of the $Q \bar{Q}$ bound state, $v=(1,0,0,0)$, and the operators $\mathcal{O}_{E}$ and $\mathcal{O}_{B}$ become

$$
\mathcal{O}_{E}=\mathbf{E}^{\mathbf{A}} \cdot \mathbf{E}^{\mathbf{A}}, \quad \mathcal{O}_{B}=\mathbf{B}^{\mathbf{A}} \cdot \mathbf{B}^{\mathbf{A}},
$$

where $\mathbf{E}^{\mathbf{A}}$ and $\mathbf{B}^{\mathbf{A}}$ are the colour electric and magnetic fields. (This explains the choice of operators and coefficients in Eq. (1).) The coefficients $c_{E}$ and $c_{B}$ can be obtained by computing the energy shift of the $Q \bar{Q}$ bound state in an external electric and magnetic field, i.e. by computing the quadratic Stark and Zeeman energies. The dominant contribution is the quadratic Stark effect, since magnetic effects are suppressed by a factor of $\vec{v} / c \approx \alpha_{s}\left(\Lambda_{Q}\right)$ relative to electric effects for each replacement $E \rightarrow B$.

The operators $\mathcal{O}_{E}$ and $\mathcal{O}_{B}$ can be written as linear combinations of the gluon energymomentum tensor,

$$
T_{\text {gluon }}^{\mu \nu}=\frac{1}{4} g^{\mu \nu} G^{\alpha \beta A} G_{\alpha \beta}^{A}-G^{\mu \alpha A} G_{\alpha}^{\nu A},
$$


and the divergence of the scale current, 2

$$
\partial^{\mu} s_{\mu}=T_{\alpha}^{\alpha}=\frac{\beta(g)}{2 g} G^{\alpha \beta A} G_{\alpha \beta}^{A} .
$$

Note that the energy-momentum tensor $T^{\mu \nu}$ that occurs in Eq. (6) is the full energymomentum tensor, whereas $T_{\text {gluon }}^{\mu \nu}$ in Eq. (5) is only the gluon contribution to the energymomentum tensor. The expressions for $\mathcal{O}_{E}$ and $\mathcal{O}_{B}$ are

$$
\begin{aligned}
& \mathcal{O}_{E}=T_{\text {gluon }}^{\mu \nu} v_{\mu} v_{\nu}-\frac{g}{2 \beta(g)} T_{\alpha}^{\alpha}, \\
& \mathcal{O}_{B}=T_{\text {gluon }}^{\mu \nu} v_{\mu} v_{\nu}+\frac{g}{2 \beta(g)} T_{\alpha}^{\alpha} .
\end{aligned}
$$

The operators $\mathcal{O}_{E}$ and $\mathcal{O}_{B}$ in the effective Lagrangian Eq. (1) are renormalised at the scale $\Lambda_{Q}$, and the operator $T_{\text {gluon }}^{\mu \nu}$ in Eq. (7) is also renormalised at $\Lambda_{Q}$. (The operator $T_{\alpha}^{\alpha}$ is scale independent.) The QCD beta function at the scale $\Lambda_{Q}$ can be written as

$$
\mu \frac{d}{d \mu} g=\beta(g)=-b_{Q} \frac{g^{3}}{16 \pi^{2}}+\ldots
$$

so that Eq. (7) can be rewritten as

$$
\begin{aligned}
& \mathcal{O}_{E}=T_{\text {gluon }}^{\mu \nu} v_{\mu} v_{\nu}+\frac{2 \pi}{b_{Q} \alpha_{s}\left(\Lambda_{Q}\right)} T_{\alpha}^{\alpha}, \\
& \mathcal{O}_{B}=T_{\text {gluon }}^{\mu \nu} v_{\mu} v_{\nu}-\frac{2 \pi}{b_{Q} \alpha_{s}\left(\Lambda_{Q}\right)} T_{\alpha}^{\alpha} .
\end{aligned}
$$

The gluon operator in Eq. (11) can be rewritten in the form

$$
c_{E} O_{E}+c_{B} O_{B}=\left(c_{E}+c_{B}\right)\left(T_{\text {gluon }}^{\mu \nu} v_{\mu} v_{\nu}\right)+\left(c_{E}-c_{B}\right) \frac{2 \pi}{b_{Q} \alpha_{s}\left(\Lambda_{Q}\right)} T_{\alpha}^{\alpha} .
$$

The matrix elements of $T_{\text {gluon }}^{\mu \nu}$ and $T_{\alpha}^{\alpha}$ in a nucleon state are known at zero momentum transfer,

$$
\begin{aligned}
\left\langle p\left|T_{\alpha}^{\alpha}\right| p\right\rangle & =2 M^{2}, \\
\left\langle p\left|T_{\text {gluon }}^{\mu \nu}\right| p\right\rangle & =2 V_{2}(\mu)\left(p^{\mu} p^{\nu}-\frac{1}{4} g^{\mu \nu} p^{2}\right),
\end{aligned}
$$

where $M$ is the nucleon mass. The value of $V_{2}$, the gluon momentum fraction in the nucleon, is measured in deep inelastic scattering, and its value can be extracted from the $F_{1}$ (or $F_{2}$ )

2 For simplicity, we will work in the limit that the light quark masses are zero. It is straightforward to include light quark mass effects $[6]$. The trick of using the scale anomaly to compute the matrix element of $G^{2}$ is due to Voloshin and Zakharov[0]. 
structure function. The usual value quoted in the literature is $V_{2}\left(\mu^{2}=16 \mathrm{GeV}^{2}\right)=0.44$ [8]. The $\mu$ dependence of $V_{2}$ may be found in [8].

The forward scattering amplitude of a $Q \bar{Q}$ meson off nucleons is completely determined in terms of the measured value of $V_{2}$,

$$
\mathcal{M}_{\mathrm{fwd}}=2 V_{2}\left(\Lambda_{Q}\right)\left(\frac{c_{E}+c_{B}}{\Lambda_{Q}^{3}}\right) M^{2}\left(\gamma^{2}-\frac{1}{4}\right)+2 M^{2}\left(\frac{c_{E}-c_{B}}{\Lambda_{Q}^{3}}\right) \frac{2 \pi}{b_{Q} \alpha_{s}\left(\Lambda_{Q}\right)}
$$

where $\gamma\left(=v^{0}\right)$ is the time dilation factor in the rest frame of the nucleon. The forward scattering amplitude is proportional to the energy shift of the $Q \bar{Q}$ meson due to its interaction with nuclear matter. The binding energy is given in terms of $\mathcal{M}$ (neglecting Fermi motion) by

$$
U_{\text {binding }}=\frac{\mathcal{M} n}{2 M}
$$

where $n$ is the number of nucleons per unit volume in nuclear matter. The factor of $2 M$ divides out the standard relativistic normalisation of nucleon states used in computing $\mathcal{M}$ in Eq. (12). The binding energy of $Q \bar{Q}$ states in nuclear matter is

$$
U_{\text {binding }}=\rho V_{2}\left(\Lambda_{Q}\right)\left(\frac{c_{E}+c_{B}}{\Lambda_{Q}^{3}}\right)\left(\gamma^{2}-\frac{1}{4}\right)+\rho\left(\frac{c_{E}-c_{B}}{\Lambda_{Q}^{3}}\right) \frac{2 \pi}{b_{Q} \alpha_{s}\left(\Lambda_{Q}\right)},
$$

where $\rho=M n$ is the mass density of nuclear matter.

To determine the value of the binding energy of a $Q \bar{Q}$ state, we need expressions for $c_{E}$ and $c_{B}$. The coefficient $c_{B}$ is suppressed relative to $c_{E}$ by a factor of $\alpha_{s}\left(\Lambda_{Q}\right)^{2}$, and can be neglected. The computation of $c_{E}$ is subtle, because gluon interactions cause mixing between the singlet and octet $Q \bar{Q}$ channels. In the heavy quark limit, $m_{Q} \rightarrow \infty$, in which the $Q \bar{Q}$ system is a Coulomb bound state, a careful derivation of $c_{E}$ has been given by Peskin [9] and we will quote his result. For the $1 s$ state, he finds

$$
\frac{c_{E}}{\Lambda_{Q}^{3}}=\frac{14 \pi}{27} r_{B}^{3}
$$

where $N_{c}$ is the number of colours, and $r_{B}$ is the Bohr radius

$$
r_{B}^{-1}=\frac{2}{3} \alpha_{s}\left(r_{B}^{-1}\right) m_{Q}
$$

Peskin used the $1 / N_{c}$ expansion in obtaining the expression for $c_{E}$ in Eq. (15). This approximation was used merely to give an expression which can be written in a simple 
form. The error in not keeping the $1 / N_{c}$ corrections was estimated to be [9] approximately $10 \%$, which is smaller than the $\alpha_{s}\left(\Lambda_{Q}\right)$ and $1 / m_{Q}$ corrections that we have neglected. Combining Eqs. (15) and (14) gives

$$
U_{\text {binding }}=\frac{14}{27} \pi r_{B}^{3} \rho\left[V_{2}\left(\Lambda_{Q}\right)\left(\gamma^{2}-\frac{1}{4}\right)+\frac{2 \pi}{b_{Q} \alpha_{s}\left(\Lambda_{Q}\right)}\right] .
$$

Treating the $\Upsilon$ as a Coulomb bound state, we find from $(16) r_{B}^{-1} \simeq 1.1 \mathrm{GeV}$. Using a more realistic potential, Quigg and Rosner [10] calculated $\langle r\rangle_{\Upsilon} \simeq 1.0 \mathrm{GeV}^{-1}$, corresponding to a Bohr radius $r_{B}^{-1} \simeq \frac{3}{2}\langle r\rangle^{-1} \simeq 1.5 \mathrm{GeV}$. To the degree to which our results are sensitive to the nonperturbative region of the potential, they are not to be trusted, and since $U_{\text {binding goes like }} r_{B}^{3}$, this introduces a large uncertainty into our results. We give an

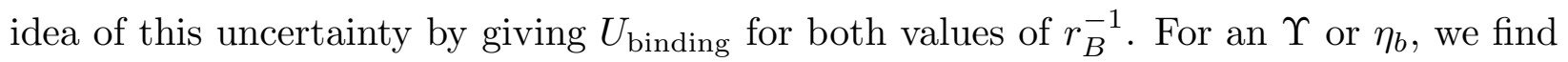
the binding energies for the state at rest in nuclear matter of

$$
\begin{aligned}
U_{\text {binding }} & \simeq 4 \mathrm{MeV}\left(r_{B}^{-1}=1.1 \mathrm{GeV}, \alpha_{s}=0.36\right) \\
& \simeq 2 \mathrm{MeV}\left(r_{B}^{-1}=1.5 \mathrm{GeV}, \alpha_{s}=0.30\right)
\end{aligned}
$$

Here we have taken $\rho=1.2 \times 10^{-3} \mathrm{GeV}^{4}$, and $b_{Q}=27 / 3$.

For the $J / \psi$, the validity of our expansion becomes markedly more suspect: from (16) we find $r_{B}^{-1} \simeq 640 \mathrm{MeV}$, while from [10] we find $r_{B}^{-1} \simeq 750 \mathrm{MeV}$, where we really do not trust our perturbative results. However, proceeding as before we find

$$
\begin{aligned}
U_{\text {binding }} & \simeq 11 \mathrm{MeV}\left(r_{B}^{-1}=640 \mathrm{MeV}, \alpha_{s}=0.6\right), \\
& \simeq 8 \mathrm{MeV}\left(r_{B}^{-1}=750 \mathrm{MeV}, \alpha_{s}=0.5\right) .
\end{aligned}
$$

Of course we do not believe this result is anything more than a rough estimate for the $J / \psi$ binding energy, as the corrections to (19) will be of order unity.

Note that our computation unambiguously determines the sign of the energy shift of $Q \bar{Q}$ mesons at rest in nuclear matter, at least in the $m_{Q} \rightarrow \infty$ limit. The spin dependent corrections are suppressed relative to Eqs. (18) and (19) by an additional factor of $\alpha_{s}$, so that the binding energy of the $\eta_{b}$ and $\Upsilon$ are approximately equal. The same arguments will also apply to the $J / \psi$ and $\eta_{c}$, but the spin symmetry breaking effects will be larger.

There are two trivial corrections to the above expression Eq. (17), which we have not included because they are small compared to the $\alpha_{s}\left(\Lambda_{Q}\right)$ and $1 / m_{Q}$ terms that we have neglected. The first is that there is a binding energy correction to the relation between the nucleon mass density, and the mass density of nuclear matter. The second is that 
the structure functions of bulk nuclear matter differ from those of nucleons, so that $\lambda$ in Eq. (17) should properly refer to the gluon distribution measured using the $F_{2}$ structure function for nuclear matter.

The results obtained in this paper show that the scattering amplitude of a $Q \bar{Q}$ meson with nuclear matter scales as $r_{Q}^{3}$, and the cross-section as $r_{Q}^{6}$ (up to the logarithmic dependence in $\alpha_{s}$ ) 3 This does not agree with the assumption in the literature that the cross-section scales like the area of the meson. We have also shown that the sign of the interaction energy is negative, so that the meson binds to nuclear matter. There are no pion corrections to our result, Eq. (17). All pion contributions are included in the matrix element of the gluon operators in nuclear matter. The only role of the pions in this calculation is to modify the density and pressure of nuclear matter. We emphasise that our results are rigorous predictions of QCD and are completely model independent in the limit $m_{Q} \rightarrow \infty$.

The largest source of error in our computation is the use of Coulomb wavefunctions to compute $c_{E}$. Non-perturbative confinement effects can be included by using a more realistic potential model to compute the bound state wavefunction. Unfortunately, the computation of $c_{E}$ involves intermediate colour octet states, and thus the $Q \bar{Q}$ Hamiltonian in the colour octet channel. In perturbation theory (the computation of Peskin [9]), one replaces the attractive $-4 \alpha_{s} / 3 r$ attractive potential in the singlet channel by a repulsive $\alpha_{s} / 6 r$ repulsive potential in the octet channel. The corresponding modification when nonperturbative effects are included in the singlet channel is more complicated, and is being investigated further.

The binding energy was computed in Eq. (17) for the $1 s$ ground state. One can compute the binding energies of the excited states using the values of $c_{E}$ given in [9] for the excited states. Since the binding energy goes like $r_{Q}^{3}$, the excited states of quarkonium will be more tightly bound in the $m_{Q} \rightarrow \infty$ limit. Naïvely applying (17) to the $\psi^{\prime}(2 s)$ gives

$$
U_{\text {binding }}^{(2 s)}=\left(\frac{502}{7}\right) U_{\text {binding }}^{(1 s)} \sim 700 \mathrm{MeV}
$$

3 The $r^{3}$ dependence of the two gauge boson-meson scattering amplitude also shows that the Rayleigh scattering cross-section is proportional to $r^{6} \omega^{4}$. The nuclear binding of $Q \bar{Q}$ mesons arises from the same operator that is responsible for Rayleigh Scattering, with the electromagnetic field strength tensor replaced by the gluon field strength tensor. 
suggesting that the $\psi^{\prime}(2 s)$ has a lower energy in nuclear matter than the ground state. The large radius of the $2 s$ is clearly an indication that the multipole expansion is breaking down, so this result cannot be trusted (but it might still be true).

We would like to thank S. Brodsky, M. Butler, D. Wasson, R. Milner, M. Wise and D. Kaplan for discussions. M. S. thanks the Institute for Nuclear Theory at the University of Washington for their hospitality while some of this work was done. As this work was being completed, we became aware of similar work in progress by J. Pasupathy [11]. This work was supported in part by DOE grant \#DE-FG03-90ER40546, and by NSF grants PHY-8958081 and PHY-9057135. 


\section{References}

[1] S.J. Brodsky, I. Schmidt and G.F. de Teramond, Phys. Rev. Lett. 64 (1990) 1011.

[2] D.A. Wasson, Phys. Rev. Lett. 67 (1991) 2237.

[3] K. Gottfried, Phys. Rev. Lett. 40 (1978) 538;

T.M. Yan, Phys. Rev. D22 (1980) 1652 ;

M. Voloshin, Nucl. Phys. B154 (1979) 365.

[4] H. Georgi, Phys. Lett. 240B (1990) 447.

[5] N. Isgur and M.B. Wise Phys. Lett. 232B (1989) 113; Phys. Lett. 237B (1990) 527.

[6] R.S. Chivukula, et al., Ann. Phys. 192 (1989) 93.

[7] M. Voloshin and V. Zakharov, Phys. Rev. Lett. 45 (1980) 688.

[8] See for example R.D. Field, Applications of Perturbative QCD, Addison-Wesley Publishing Company, 1989.

[9] M.E. Peskin, Nucl. Phys. B156 (1979) 365.

[10] C. Quigg and J. L. Rosner, Phys. Rev. D23 (1981) 2625.

[11] J. Pasupathy, private communication. 